\title{
L'opérationnalisation Des Politiques Publiques Face A La Négociation Et La Marchandisation Politiques Au Bénin De 2006 A 2016
}

\author{
Victorin Vidjannagni Gbenou (Maître Assistant) \\ Département de Sociologie-Anthropologie, \\ Université d'Abomey-Calavi, Bénin
}

Doi:10.19044/esj.2018.v14n35p201 URL:http://dx.doi.org/10.19044/esj.2018.v14n35p201

\begin{abstract}
This paper focuses on the politicization of the operationalization of public policies in Benin from 2006 to 2016. Here, the phenomenon has been accentuated with the direct consequence of questioning the relevance of public action. Its overall objective is to analyze the political practices that have supported the operationalization of public policies from 2006 to 2016. In answering the research question asked, the variables generated by the working hypotheses are related to political negotiation, political haggling, and political blackmail. The research is anchored on the theoretical current interactionist. It is both qualitative and quantitative at the same time. The data were collected from stakeholders involved in the process in Cotonou, Porto-Novo, and Parakou from September 2017 to June 2018. The sample consists of 247 actors (16 for interviews, 231 informants per questionnaires). Informants were selected using reasoned choice and random choice after Schwartz's formula. The analysis of the result shows that political negotiation requires promises around public policies to be more conclusive while relying on the various lobbies. Political commodification leads to an open market on which public markets, business, posts and political investments, and public policies are found. Political blackmail is being implemented at the time based on the operationalization of public policies. Political support is a guarantee and the outcome of public policies is significantly dependent on the vote.
\end{abstract}

Keywords: Operationalization, negotiation, commodification, public policy, Benin

Résumé

La présente recherche traite de la politisation de l'opérationnalisation des politiques publiques au Bénin de 2006 à 2016, pays où le phénomène a été accentué, avec pour conséquence directe, la remise en cause de la pertinence 
de l'action publique. Elle s'est fixée comme objectif global d'analyser les pratiques politiques ayant soutenu l'opérationnalisation des politiques publiques de 2006 à 2016. En répondant à la question de recherche posée, les variables générées par les hypothèses de travail sont relatives à la négociation politique, au marchandage politique et au chantage politique. La recherche est ancrée dans le courant théorique interactionniste. Elle est qualitative et quantitative à la fois. Les données ont été collectées auprès des acteurs impliqués dans le processus à Cotonou, Porto-Novo et Parakou de septembre 2017 à juin 2018. L'échantillon est constitué de 247 acteurs (16 pour les entretiens, 231 informateurs par questionnaire). Les informateurs ont été sélectionnés à l'aide des techniques de choix raisonné et de choix randomisé après l'application de la formule de Schwartz. Il ressort de l'analyse des résultats que la négociation politique nécessite des promesses autour des politiques publiques pour être plus concluante tout en s'appuyant sur les divers lobbys. La marchandisation politique entraîne un marché ouvert sur lequel il est retrouvé les marchés publics, des affaires, des postes et placements politiques et les politiques publiques. Le chantage politique se met en œuvre à l'heure de l'opérationnalisation des politiques publiques. Le soutien politique constitue un gage et l'aboutissement des politiques publiques dépend du vote.

Mots-clés : Opérationnalisation, négociation, marchandisation, politique publique, Bénin

\section{Introduction}

Dans tout régime démocratique, les élections (surtout présidentielles) donnent lieu à une « compétition bien organisée » (Champagne, 2001) et non à une simple joute électorale où ce qui pourrait contribuer à l'emporter pourrait être en décalage vis-à-vis des aspirations profondes et besoins du peuple souverain. Cette considération de départ sous-entend que le fait majeur qui fonde et justifie la démocratie est que le " pouvoir appartient au peuple. C'est lui [le peuple] qui le donne à des autorités démocratiquement élues et installées pour qu'elles l'exercent au nom du peuple, avec lui, par lui et pour lui. » (Lapierre, 2002, p.88). Ainsi, tout dirigeant doit son pouvoir à son peuple.

Dans un régime démocratique, l'élection d'une personnalité politique est conditionnée par la qualité, la pertinence et la cohérence de son projet de société. Autrement dit, aucun candidat à une élection présidentielle ne devrait être élu à aucune fonction républicaine s'il n'est pas porteur d'un projet de société ou agenda public. Dans ce sens, Banégas affirme :

«Les joutes électorales ou compétitions politiques ont lieu

autour des idées contenues dans les projets de société des 
candidats. L'idéal serait que les candidats élus soient ceux et celles qui portent les projets de société les plus pertinents, c'est-à-dire les agendas politiques qui répondent le mieux aux besoins et aspirations du peuple » (Banégas, 2000, p.37).

Sur la base de cette considération, il convient d'en déduire que le « contrat politique public » qui lie une autorité élue à ses mandants et au peuple tout entier est le projet de société. Aussi, se dégage-t-il une première interrogation fondée : qu'est-ce qu'un projet de société et pourquoi est-il si important dans un régime démocratique ? C'est une considération de départ à mentionner à toutes fins utiles. Choisissant de ne pas verser dans une littérature redondante et inutile parce qu'elle est abstraite, il faut couper court en voyant simplement le projet de société comme le contrat public qui lie une personnalité publique candidate élue à ses mandants et surtout à son peuple. Le projet de société n'est pas un document de planification du développement, ce n'est pas un catalogue d'intentions crues ou un recueil de rêves portés par un vil aspirant au fauteuil présidentiel. En réalité, le projet de société est le contrat politique entre un candidat à une élection et le peuple qu'il aspire diriger. Pour Braud, «Un projet de société est un engagement sérieux que prend un candidat à élection politique. Il est un cahier de charges formel, s'il faut emprunter la logique démocratique qui priorise le fait que le peuple est l'unique détenteur du pouvoir. C'est pour cela que la conception et l'élaboration d'un projet de société se font toujours en parfaite adhésion avec le peuple suivant une démarche participative dans un régime démocratique » (Braud, 2003, p.51-52).

Lorsqu'un Président de la République est élu dans les conditions préalablement décrites, il s'appuie sur trois principes fondamentaux en vue de l'Action Gouvernementale : 1) la constitution de la République pour ne jamais transgresser la loi et parjurer, 2) la vision nationale de développement pour ne pas s'écarter du chemin déjà tracé avant son arrivée, et 3) son projet de société qui contient sa vision en vue de tenir ses engagements. Ledit projet doit également tenir compte de la dynamique du moment dans la mesure où un projet de société peut s'inscrire dans la continuité mais doit aussi innover par une rupture avec un ordre ancien inadapté aux réalités du moment dans la logique de mieux faire. C'est cette logique qui sous-tend et oriente l'action publique qui doit être une réponse à la demande populaire depuis la base.

Le Chef de 1 'État est donc comme un soumissionnaire que le peuple recrute après à un appel à candidature (élection) pour exécuter une tâche (commande publique) dont il a pris officiellement l'engagement (contrat). Cet éclairage donne lieu à une deuxième interrogation majeure : les Présidents de la République élus au Bénin sont-ils conscients qu'ils sont commis 
ponctuellement par le peuple pour l'exécution du chantier du développement national ?

En observant de près la gouvernance politique et la pratique politique, l'exercice du pouvoir politique par le Chef de l'État au Bénin de 2006 à 2016, il est évident qu'il se comporte comme celui qui rend service au peuple. Il se comporte comme un maître d'œuvre qui commande et exige. Il se trouve que les rôles semblent être inversés au regard d'un certain nombre de faits palpables. Le Chef de l'État se comporte tout comme si c'est lui qui a commis le peuple. Cette situation mérite d'être profondément analysée. C'est d'ailleurs la trame de cette production scientifique.

Afin d'analyser l'opérationnalisation des politiques publiques face à la négociation et la marchandisation politiques au Bénin de 2006 à 2016, l'analyse des variables considérées se fera en trois principaux mouvements : i) l'opérationnalisation des politiques publiques comme terme de négociation politique, ii) l'opérationnalisation des politiques publiques comme instrument de marchandisation politique et iii) l'opérationnalisation des politiques publiques comme outil de chantage politique.

\section{PRÉCISIONS THÉORIQUE, CONCEPTUELLE ET MÉTHODOLOGIQUE}

\subsection{Précision théorique}

Suite aux constats faits, le problème traité dans cet article scientifique est la «politisation de l'opérationnalisation des politiques publiques au Bénin de 2006 à 2016 ». Ce problème a pour conséquence directe : la «remise en cause de la pertinence de l'action publique du développement au Bénin de 2006 à 2016 ». Il est bien logique que lorsque l'opérationnalisation des politiques publique est politisée, cela engendre des frustrations qui suscitent beaucoup d'interrogations sur la pertinence de l'action publique du développement. Dans ce sens, la question de recherche posée est : «comment l'opérationnalisation des politiques publiques a été politisée au Bénin de 2006 à 2016 ? ». L'opérationnalisation de cette question de recherche fait intervenir des variables.

En fait, la variable maîtresse ou variable principale de cette recherche s'énonce : « opérationnalisation des politiques publiques 》. Cette variable est désagrégée en trois (3) sous variables qui sont : 1) «négociation politique », 2) « marchandage politique » et 3) «chantage politique ». Ces trois variables secondaires dans la hiérarchie et norme des hypothèses, sont mobiles ou inconstantes. Elles se répercutent également dans les objectifs de la recherche telle que fixée.

Il est important de mentionner que l'action publique n'est pas détachée totalement de l'action politique (Ibid.3). Dans ce sens, la " négociation politique " intervient pour contribuer à ratisser large dans la mobilisation 
électorale. Pour Bourdieu (1981), la négociation politique pose problème lorsqu'elle est politisée puisqu'elle n'est plus normale telle que règlementée. Par ailleurs, selon Chevalier (2005), le « marché politique » peut accélérer et rendre efficace le service public. Dans ce cas, ce n'est pas un vain marchandage politique. Par contre, la " marchandisation politique nuisible » évoquée par Banégas (1998) est la «marchandisation du vote ». L'expression du suffrage universel est liée à la "corruption politique » qui a lieu à travers une avance en vue du vote. Une fois accédé au pouvoir, l'homme politique qui n'a pas tous les moyens pour satisfaire les déshydratas de ses anciens électeurs procède par «chantage politique » (Ibid.3).

En termes de modèle d'analyse, cette recherche est inscrite dans le courant interactionniste à travers le paradigme «champ politique» de Bourdieu (1981) et la théorie des «fonctions politiques» de Duverger (2000). Ces deux théories ont servi à analyser les résultats générés par la recherche.

\subsection{Description de l'approche méthodologique}

Cette recherche a fait intervenir aussi bien des données quantitatives que des données qualitatives, compte tenu des exigences méthodologiques du sujet traité qui est relatif à l'opérationnalisation des politiques publiques au Bénin entre 2006 et 2016. Cinq (5) principales catégories d'acteurs sont impliquées dans la recherche: 1) animateurs politiques, 2) les animateurs des Organisations Non Gouvernementales locales, 3) universitaires et scientifiques, 4) cadres des administrations et 5) militants et partisans politiques. Ces acteurs ont été échantillonnés suivant un protocole mixte : méthode de saturation pour l'enquête par entretien (16 entretiens) et l'application de la formule de Schwartz en ce qui concerne l'enquête par questionnaire (231 informateurs). Ainsi, la taille de l'échantillon est de 247 acteurs choisis selon les techniques de choix raisonné et de choix randomisé.

Quant à la collecte des données, elle a lieu suivant l'enquête par entretien et l'enquête par questionnaire. L'enquête par entretien a fait intervenir l'entretien individuel semi-structuré, l'entretien collectif structuré et la discussion de focus groupe. Le guide d'entretien a été spécifié par rapport aux cibles. En ce qui concerne l'enquête par questionnaire, il faut préciser que le questionnaire a aussi été spécifié par rapport aux cibles d'informateurs. La première phase a été celle de la pré-enquête qui a duré une vingtaine de jours (du 3 au 27 juin 2017). Cette phase a permis de tester les outils de collecte en vue de les réajuster. L'enquête proprement dite a eu lieu de façon discontinue de septembre 2017 à juin 2018, avec des séjours d'au moins deux mois continus activement passés sur le terrain de manière à collecter les données auprès des cibles visées. Les instruments traditionnels de collecte de données (papier, stylos, enregistreur, appareil photographique, etc.) ont été mis à contribution. 
Pour ce qui est du traitement des données collectées, il est important de mentionner que le travail a été fait en équipe. Les données qualitatives ont été transcrites et stockées. Quant aux données quantitatives, elles ont été directement stockées. Il s'en est suivi l'apurement, le traitement, le dépouillement, la catégorisation, l'analyse et l'interprétation. Pour faire ce travail, les logiciels World, Excel, SPSS et Epi-info ont été utilisés.

Par ailleurs, il est important de souligner que les contraintes survenues sur le terrain lors de la collecte des données sont d'ordre structurel, organisationnel et beaucoup d'entre elles dues à des tendances lourdes humainement crées. L'expérience de terrain a permis d'y apporter des solutions.

\subsection{Description du cadre de la recherche}

La recherche faite dans le cadre de la rédaction de cet article scientifique a lieu dans les trois (3) communes à statut particulier au Bénin : Cotonou, Porto-Novo et Parakou. Traitant d'une question nationale, le choix de ces trois communes ne s'est pas fait au hasard.

La commune de Cotonou est la plus grande métropole du Bénin. Aujourd'hui, la population de Cotonou avoisine le million d'habitants (679 012 habitants en 2013). Selon la même source, la commune de Cotonou dispose d'une population électorale estimée à 328000 électeurs. Cette population va grandissante d'année en année dans la mesure où les citoyens en âge de voter qui entrent dépassent de loin ceux qui y sortent pour cause de décès. Politiquement conçu, la fièvre politique nationale commence souvent par Cotonou. Cela s'explique par plusieurs facteurs. En tant que première ville du Bénin, Cotonou draine une forte dynamique politique. Il serait quasiment impossible d'être élu président de la République sans faire au moins une performance électorale de $30 \%$ des suffrages électoraux. Cette même dynamique électorale drainée par Cotonou est directement répercutée sur les communes voisines qui entrent dans la «danse électorale» (Danioue, 1997) selon la fièvre électorale à Cotonou, ville d'opposition ou de mouvance.

Quant à la commune de Porto-Novo, elle est peuplée de 264320 habitants en 2013 contre 223552 en 2002. La population électorale de PortoNovo est estimée à 184223 électeurs. Politiquement, Porto-Novo s'illustre par son histoire politique. Porto-Novo est la cité natale du feu président Sourou Migan Apity qui, à l'aide du Parti Républicain du Dahomey (PRD), a pris activement part à l'animation politique nationale du Dahomey au Bénin. La fièvre électorale de Porto-Novo conditionne la tendance politique de l'Ouémé et du Plateau. Porto-Novo est la capitale administrative du Bénin. Il est généralement conçu que Porto-Novo est politiquement très fidèle.

La commune de Parakou est peuplée de 255478 habitants en 2013 contre 149819 habitants en 2002. Elle est la porte d'entrée du grand 
septentrion (Alibori, Atacora, Borgou, Collines et Donga). Parakou est le fief traditionnel du feu président Hubert Coutoucou Maga, premier président du Bénin. La population électorale de Parakou est estimée à 171528 électeurs, avec une forte communauté venue du Sud qui s'organise pour prendre leur place au sein de l'arène politique. Leur implication est déterminante dans l'issue d'un vote, d'où l'adoption d'une stratégie de positionnement.

La période allant de 2006 à 2016 a été choisie simplement parce que le phénomène de politisation des politiques publiques au Bénin était très accentué et perceptible. Il convient de mentionner que la gouvernance politique de l'époque tenait assez compte des mouvements et manifestations politiques. Le Chef de l'État d'alors était souvent appelé «au secours » par les manifestants politiques préoccupés par le développement de leur localité et non forcément de tout le pays. Cette situation rejoint la tendance décrite par Braud (1996) en évoquant l'émotion en politique.

\section{ANALYSE ET DISCUSSION}

\subsection{Négociation politique}

La négociation politique s'affirme par 1) la recherche des termes de négociation, 2) les canaux de négociation politique et 3) le résultat de la négociation politique.

\subsubsection{Termes de négociation politique}

Pour réussir une négociation politique, il faut préalablement identifier les termes de la négociation. Pour le faire, les acteurs politiques mettent premièrement en avant les politiques publiques. Dans ce cadre, l'enquête de terrain a révélé les principaux secteurs pourvoyeurs de thèmes de négociation politique. La Figure 1 présente la distribution relative aux termes de négociation politique.

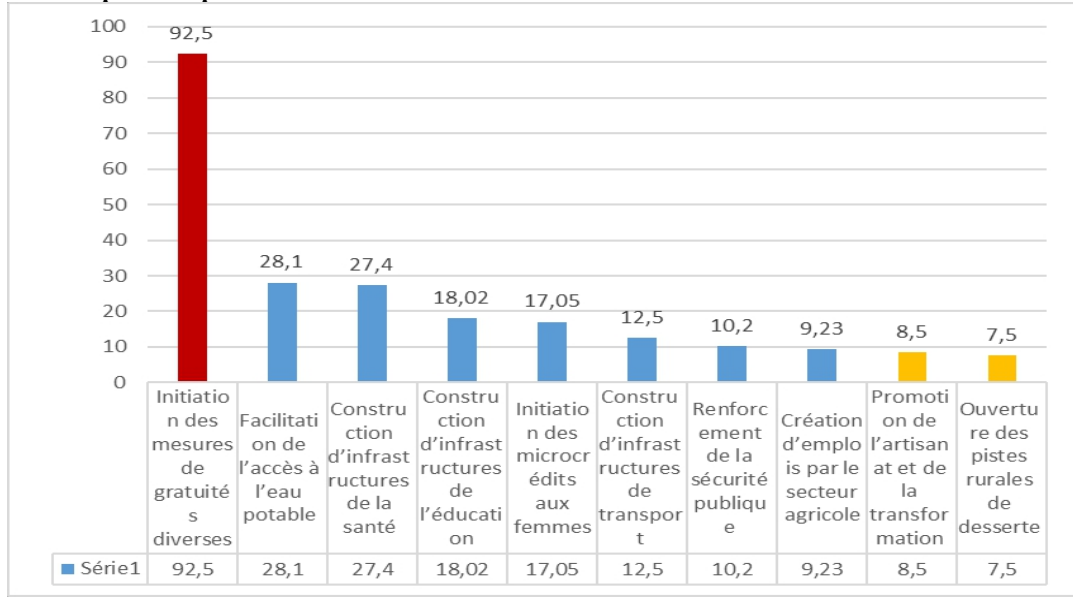

Figure 1. Termes de négociation politique et leur importance Source : données d'enquête, janvier- juin 2018 
Il ressort de la Figure 1 que les populations sont préoccupées par des mesures de gratuités diverses puisque cette tendance s'exprime à $92,3 \%$ des enquêtés au sein des dix (10) thèmes de négociation politique les plus utilisés par les acteurs politiques. Par contre, les populations sont moins préoccupées par la promotion de l'artisanat $(8,5 \%)$ et l'ouverture de pistes de desserte rurale $(7,5 \%)$. Ces différentes variables sont celles sur lesquelles les acteurs politiques à la recherche de pouvoir agissent pour négocier le vote des populations.

\subsubsection{Canaux de négociation politique}

La négociation politique est mise en œuvre à partir de plusieurs canaux qui s'expriment diversement face au fait politique global au Bénin de 2006 à 2016. En termes clairs, pour négocier politiquement il faut agir sur ce qui rend sensible l'électeur pour qu'il cède face à la négociation. Pour Courty (2006), la négociation politique est souvent conduite par des groupes d'intérêt. Ils agissent ainsi parce qu'ils ont des intérêts à protéger. Ces lobbys sont souvent contrecarrés par des « acteurs pragmatiques pour le changement » (CAP). Les acteurs interrogés ont déclaré implicitement l'existence de plusieurs canaux de négociation politique :

\section{- Lobbys religieux}

Entre 2006 et 2016, les lobbys religieux étaient très présents dans la négociation politique. Les fidèles de tous bords religieux mais surtout chrétiens évangélistes sont sensibilisés politiquement pour soutenir le pouvoir en place. À ce propos, un des acteurs interrogés affirme :

"Jusqu'à un passé récent, on a vu dans ce pays des pasteurs devenir des hommes politiques, des hommes d'affaires, des commerçants et même des chefs de partis politiques. Ce sont eux qu'on envoie aller sensibiliser les gens pour le compte du Patron. Il était très difficile d'obtenir quelque chose dans ce pays sans prendre par eux. Très souvent, on vous demande qui vous a envoyé et quelle est votre église. Mais personne ne vous demande ce que vous savez faire. Au nom du lobbying religieux politique, on a $v u$ des gens sans aucune compétence, sans aucun savoir-faire devenir ministre du jour au lendemain et narguaient tout le monde. "

\section{(Dirigeant d'ONG. Cotonou, 21/01/2018)}

Cela prouve bien la force des lobbys religieux en politique. C'est sur cet atout que beaucoup de négociations politiques ont été faites et ont connu un succès certain. Les leaders politiques demandaient souvent de réunir 
le "corps du Christ pour une importante communication". C'est un mot d'ordre qui est ainsi lancé et qui met en mouvement l'action politique.

- Lobbys ethniques et régionaux

Au Bénin, en politique, l'ethnie et la région comptent beaucoup. Jusque qu'à ce jour, il est impossible de se faire propulser au pouvoir sans l'appui d'un groupe politique ou d'une région. Lorsque la variable ethnique ou socioculturelle s'introduit dans le processus de négociation politique, elle devient même déterminante. Ceci revient à dire que le cautionnement ethnique a de la valeur en politique. C'est cette considération qui fait la trame de l'analyse de Bako Arifari (1995) lorsqu'il évoque la « logique du fils du terroir face au vote et au fait politique ». Telle est aussi la préoccupation de Bourdieu (1981) qui évoque la conscience de la « source politique », la «racine politique » et le «terroir politique ». Les lobbys ethniques constituent la première source de pouvoir. Un acteur interrogé à ce propos a déclaré ce qui suit :

"Chez nous ici, c'est notre frère que nous connaissons. C'est le sang que nous votons. On peut tout dire, nous sommes comme ça. Celui qui donne la preuve qu'il est d'ici a déjà gagné notre confiance à $50 \%$. Le reste, il viendra négocier et on lui dira comment ça se passe et ce qu'il doit faire. Ce qui est sûr, il faut être d'abord d'ici ou de proche et après vous allez nous promettre l'eau, l'électricité, les emplois et des écoles et on va voter pour vous. Ce n'est donc pas gratuit. Il y a une légère contrepartie. "

(Militant de parti politique. Parakou, 17/12/2017)

\section{- Lobbys des affaires}

Le lobby des affaires est un lobby très puissant. Ce lobby a pour terreau l'argent et l'influence. Le lobby des affaires est très efficace dans la négociation politique car il négocie avec l'argent qui est déterminant au sein de l'arène politique. La mise en œuvre des politiques publiques est aussi une promesse des hommes riches qui profitent de ce fait pour négocier l'obtention du pouvoir et le maintien au pouvoir politique. Un acteur appartenant à ce lobby et ayant été interrogé affirme :

"Le lobby des affaires est un lobby très puissant. Nous avons les moyens parce que nous sommes des hommes d'affaires pour la plupart. Nous appuyons les hommes politiques à venir au pouvoir et eux, ils nous font le retour. Alors quand quelqu'un veut être candidat, nous évaluons ses chances. S'il a beaucoup de chance, nous le soutenons en faisant les 
négociations pour lui. Dans ces négociations, nous l'aidons à faire des réalisations: matches de football, forages d'eau, construction de salles de classes, dons aux groupements féminins, dons aux écoles et hôpitaux, etc. Très souvent, les candidats que nous soutenons gagnent. ».

\section{(Homme d'affaires engagé en politique. Cotonou,} 17/12/2017)

\section{- Lobbys des associations diverses}

Dans le but de se hisser au pouvoir, les acteurs politiques militent au sein de plusieurs organisations non politiques relevant de la société civile. Ces organisations leur servent après de cadres et de tremplins pour la négociation politique. Ils agissent donc par le truchement de ces organisations pour négocier le pouvoir politique. Dans la balance, il est mis la réalisation des politiques publiques et la réponse à des demandes qui ne sont pas individuelles mais plutôt collectives. Les associations sont des tremplins de quêtes en vue du développement local. Interrogé sur cette question, un acteur politique dévoile une partie de sa stratégie politique en ces termes :

«Je suis membre de plusieurs organisations qui n'ont rien à voir avec la politique. Je suis membre de plusieurs associations de développement, des associations religieuses de mon église, des associations sportives, des associations d'activités littéraires. Rien que pour leur entretien, je sais ce que je dépense par mois. Pour avoir leurs suffrages, j'ai dû construire un pont, un module de trois salles de classes et un forage. C'est suite à cela que j'ai démontré ma capacité à répondre aux demandes des populations à la base. Celui qui ne peut pas répondre à la demande des communautés, ne peut pas être élu facilement député. Ce sera très difficile ici. »

(Député à l'Assemblée Nationale. Porto-Novo, 7/02/2018)

\section{- Lobbys corporatistes}

En négociation politique, les lobbys corporatistes sont très actifs au Bénin. Muller appelle lobby corporatiste, « toute organisation ou regroupement selon la corporation, la profession ou le métier et qui agit en vue d'un objectif bien précis. Les travailleurs d'une même organisation ne constituent pas un lobby pour rien. Si ce n'est pas pour agir pour le syndicalisme alors c'est pour agir pour la politique» (Muller, 2000, p.34). Dans ce cadre, les lobbys corporatistes agissent d'abord dans leurs milieux professionnels et après dans toute la société. Dans cette branche, les négociations politiques prennent en 
compte des politiques publiques ayant trait au travail. Un acteur interrogé à ce propos a déclaré ce qui suit :

« Nous avons voté pour un des nôtres parce qu'il nous a rassuré sur plusieurs points. Pour nous, il est question d'avoir de meilleures conditions de vie et de travail. Le camarade en question a compris puisqu'il est des nôtres et a été même patron de syndicat. Il nous représente à l'Assemblé Nationale. Nous verrons bien ce que ça va donner. Désormais, nous avons quelqu'un là-bas qui veille sur nos intérêts. »

(Dirigeant syndical. Cotonou, 6/03/2018)

\section{- Lobbys Familiaux}

Contrairement à ce que l'on peut croire, les lobbys familiaux sont très exigeants. Les exigences ne sont pas seulement relatives à la famille. Elles le sont également à la ruelle et au quartier. En dehors des cas personnels qui sont réglés, il faut aussi apporter de l'eau dans la rue, l'électricité dans la localité, réaliser une petite passerelle ou subventionner les études de deux ou trois étudiants originaires du quartier ; bien entendu que tout cela doit partir de la famille vers les autres. Les lobbys familiaux ne drainent pas de monde mais ils sont très efficaces car ils sont très fidèles.

En s'intéressant à la sollicitation et à l'efficacité des divers lobbys susévoqués, il a été remarqué que tous les lobbys ne sont pas actifs et opérationnels de la même façon et avec la même efficacité. En fait, le choix d'un lobby dépend essentiellement de trois facteurs principaux selon Polin (2007). Un homme politique qui voudra choisir un ou plusieurs canaux pour exprimer sa capacité d'action tiendra compte de : 1) moyens financiers disposés, 2) sphère de popularité et de forte audience et 3) cible (électorat) à convaincre en fonction des capacités et des efforts déployés. Suivant cette considération qui a été prise en compte très tôt dans la collecte des données, il a été observé les tendances diverses présentées par la Figure 2 qui suit :

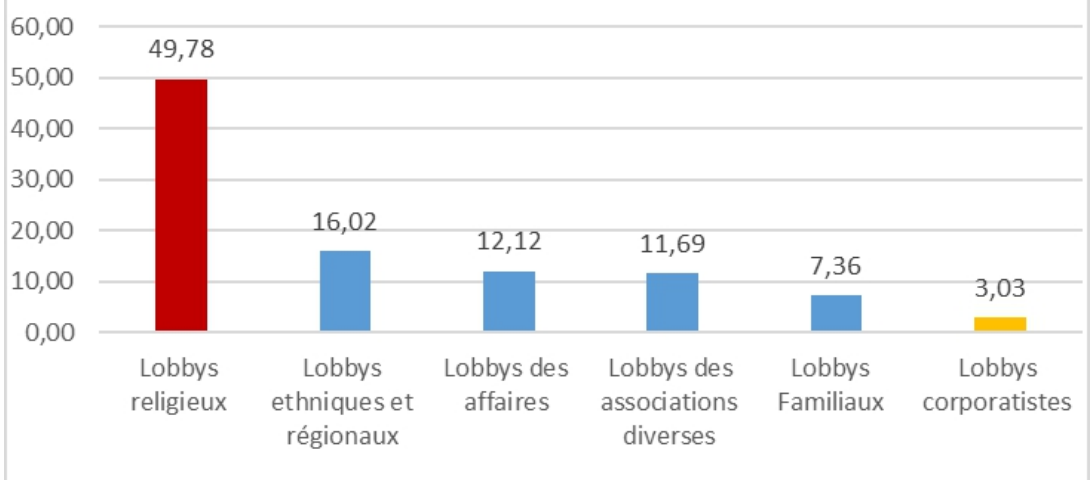

Figure 2. Tendances et expression des canaux de négociation politique Source : données d'enquête, janvier - juin 2018 
Il ressort de la Figure 2 qu'entre 2006 et 2016, les lobbys religieux ont été plus actifs dans la négociation du pouvoir (49,78\%). Cela se confirme par le fait que l'ensemble des fidèles politiquement engagés étaient appelés "Corps du Christ » qui était souvent réuni et consulté. Les lobbys ethniques et régionaux viennent ensuite (16,02\%). Cela s'explique par le fait que depuis toujours, la promotion politique a été adossée à «l'ethnisation » du combat politique. Les partis politiques sont, pour la plupart, ethniques à allure de clubs électoraux plutôt que des véritables partis politiques nationaux. Quant aux lobbys corporatistes, ils sont les moins actifs $(3,03 \%)$ parce que les acteurs appartenant à une même corporation sont différemment engagés en politique.

\subsection{Marchandisation politique}

Au Bénin, en 2006 et 2016, la marchandisation politique s'est exprimée à travers deux canaux principaux: 1) le marché des politiques publiques et 2) le marché des retombée politiques.

\subsubsection{Marché autour des politiques publiques}

L'accession au pouvoir d'État a toujours une contrepartie à payer en nature ou par divers modes mis en œuvre avant, pendant ou après l'accession au pouvoir. Toutefois, il faut préciser que les communautés exigent que toutes les négociations soient faites avant le vote car selon elles, "Les promesses politiques n'engagent que ceux qui y croient. C'est pour cette raison que l'on préfère que les choses soient traitées ici et maintenant. Après on ne les voit plus ! ». Il s'agit là d'une logique de prudence et de programmation guidée par le dépôt d'une caution non formelle (Dupy-Dery).

Il importe de faire premièrement la nuance entre "marchandisation politique" et "marchandisation du vote". Selon Largoye, «La marchandisation politique consiste à convertir en marché tout ce qui a trait à la politique comme l'art de gérer la cité. Dans ce cas, on vend et on achète sur le marché politique des idées, des astuces, des acteurs, des institutions, de l'argent, etc. " (Largoye, 2008, p.39). Par contre, selon Banegas (1998), la marchandisation du vote consiste à transformer le vote en un marché. Sur ce marché, on n'achète que le suffrage électoral à cause de la faim, du ventre et de l'argent. La marchandisation du vote ne concerne que le vote.

Sur cette base, il a été observé que les politiques publiques sont utilisées comme marché pour accéder au pouvoir politique. La patronymie, les relations, l'ancienneté, etc. ne suffisent plus pour convaincre les populations à la base. Surtout dans le cadre d'une élection présidentielle, les communautés à la base exigent un "contrat de développement" avec les candidats à l'élection présidentielle. Seuls les candidats ayant accepté de signer un contrat de développement avec la base sont pris au sérieux. C'est aussi évident que 
c'est un tremplin de rançonnement des candidats. Qu'est-ce que le contrat de développement dont il est question en réalité ?

Le contrat de développement exigé par les populations pour accorder leurs suffrages aux candidats en lice est un simple catalogue de politiques publiques ou de demandes ayant trait à la vie de toute la communauté. Généralement, il est question d'adduction d'eau potable, de pistes rurales de desserte, d'infrastructures sociocommunautaires de développement, d'appui à l'éducation, de micro finances, de problèmes de fonctionnement de centres de santé, etc.

En fait, le marché autour des politiques publiques fonctionne de façon très particulière. Ce marché politique intègre trois principales variables qui constituent les déterminants de succès ou d'échec de la marchandisation politique : 1) les politiques publiques, 2) les postes et placements politiques et 3 ) les marchés publics et affaires. Ces trois déterminants n'ont pas eu la même valeur entre 2006 et 2016 . Les données relatives à cette variable se présentent comme suit :

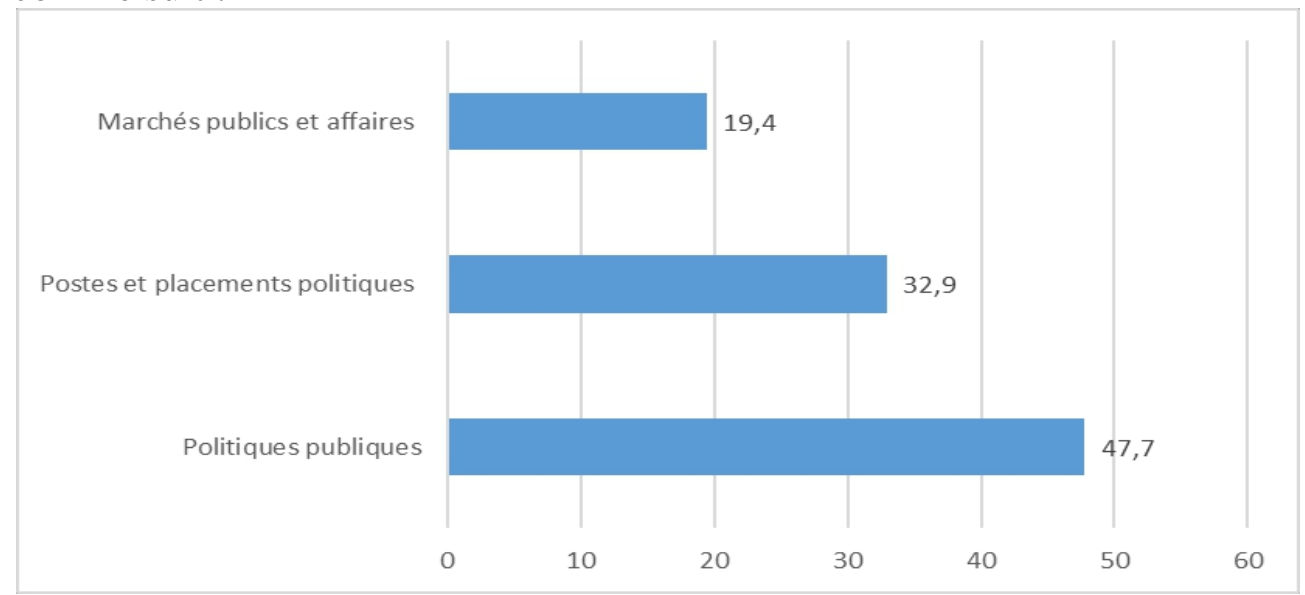

Figure 3. Offres et produits du marché politique

Source : données d'enquête, janvier-juin 2018

La Figure 3 montre clairement que le marché politique au Bénin de 2006 à 2016 était animé par trois produits principaux dont les politiques publiques constituent le produit le plus important. Pour négocier un marché, les politiques publiques constituent la première préoccupation de la marchandisation politique. Cette tendance a été confirmée par les propos des acteurs ayant été interrogés. À ce propos, un acteur politique affirme :

"Lorsque vous ambitionnez le pouvoir politique dans ce pays et surtout le poste de président de la République, vous devez faire un marché avec ceux qui vont vous soutenir. Ce marché prend en compte essentiellement les réalisations à faire dans les 
localités, les cadres à placer à divers postes politiques et techniques et les marchés publics que vous devez céder aux hommes d'affaires. Sans ces trois choses, vous n'aurez rien. Des gens sont venus ici et ont pris des engagements, mais par la suite ... !

(Acteur politique. Parakou, 12/03/2018)

\subsubsection{Marché autour des retombées politiques}

Les retombées politiques constituent l'ensemble de tous les avantages dont bénéficient les soutiens d'une personnalité politique élue à une fonction publique. Pour Genieys (2011), le soutien politique n'est pas univoque ; il est relatif à celui qui soutient et à ses ambitions. En politique, le soutien n'est pas désintéressé. Le soutien est fait des retombées politiques. Les raisons pour lesquelles les élites soutiennent ne sont pas les mêmes que celles pour lesquelles la masse ou les hommes d'affaires soutiennent. Chaque cible a ses intérêts. Il est vrai que les politiques publiques sont au cœur des raisons de soutien de part et d'autre mais diversement exprimées dans des tendances très différentes. La Figure 4 montre l'importance des politiques publiques dans les retombées politiques à l'endroit des soutiens politiques.

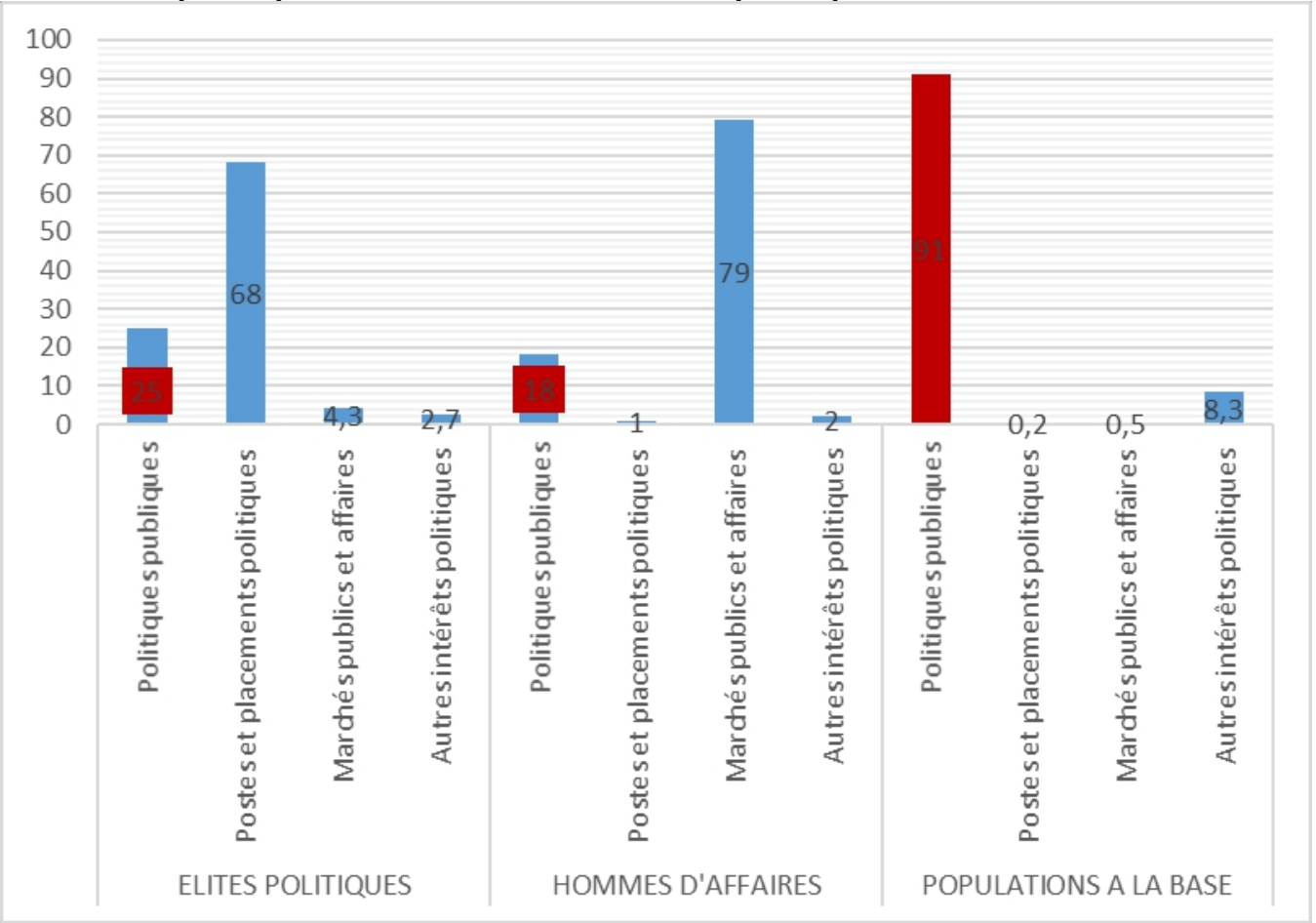

Figure 4. Importance des catégories de retombées politiques pour chaque cible Source : données d'enquête, janvier - juin 2018 
L'analyse de la Figure 4 montre clairement que les politiques publiques sont toujours au cœur du soutien politique. C'est la première retombée politique lorsque le soutien est fait à un candidat à une fonction politique. Toutefois, il faut préciser que les politiques publiques comme retombée politique sont d'abord l'affaire des populations (91\%), les élites politiques en sont légèrement préoccupées $(25 \%)$ et les hommes d'affaires n'en sont pas tellement préoccupés $(18 \%)$. C'est donc une réalité que les politiques publiques constituent la première retombée politique en termes de marché politique. Après tout, les politiques publiques contribuent au mieuxêtre et au développement.

\subsection{Chantage politique}

Dans la période sous revue $(2006$ - 2016), le chantage politique concernait étroitement les politiques publiques et surtout le processus de la mise à l'agenda politique.

\subsubsection{Chantage politique par le canal des politiques publiques}

En politique, le chantage est une réalité évidente. Genieys perçoit le chantage politique comme "[...] il est le moyen (stratégie) par lequel les acteurs politiques agissent sur les leviers déterminant l'accession ou la conservation du pouvoir politique. Le vote, les postes, les marchés, les gains, les retombées sont conditionnés par des demandes plus ou moins fortes " (Genieys, 2012, p.23).

Entre 2006 et 2016, le chantage politique a été fortement utilisé. Le recours au chantage a pris de l'ampleur surtout à partir de 2007 aussi bien au cours des élections législatives, communales, municipales que présidentielles. C'était un véritable chantage qui était fait aux populations, parfois même des menaces voilées. Les acteurs votants interrogés ont tenu les propos suivants : "Ce qu'on a enduré à la vieille de l'élection présidentielle était terrible. On n'a jamais vécu ça ici. La voie que vous voyez-là maintenant était raclée complètement deux semaines avant la campagne électorale. Plusieurs autorités sont venues ici et nous ont dit que si on ne vote pas pour la liste du Chef de l'État que les travaux seront abandonnés et qu'ils ne reviendront jamais là-dessus. On n'avait pas le choix. On a fini par voter ce qu'ils ont voulu qu'on vote. Vous-mêmes, vous voyez bien que jusque-là, les travaux ne sont pas encore achevés. C'est ça la logique politique. Ils vont vous promettre tout mais au finish, vous n'aurez rien!"

(Enseignant à la retraite. Porto-Novo, 23/04/2018) 
À sa suite, un autre acteur de la société civile membre de l'association de développement d'une localité a confié, dans un état d'âme particulier, ce qui suit :

"A la veille de l'élection présidentielle, ils sont venus ici nous dire de voter pour le candidat du Chef de l'État sinon nous n'aurons pas l'électricité. Or, ils ont déposé les poteaux électriques ici depuis plus de deux ans. On n'a vu personne. On s'est rapproché du Maire et il nous a conviés à la patience. En fin de compte, on vient conditionner l'avancée des travaux par nos votes. Donc si on ne vote pas, on n'aura rien. Pourquoi on élit un Chef d'État? N'est-ce pas pour qu'il travaille? [...]»

(Acteur de la société civile. Parakou, 7/05/2018)

Par ailleurs, il convient de mentionner que pour réussir le chantage politique, ce ne sont pas toutes les politiques publiques qui sont mises à contribution. Il y en a qui préoccupent particulièrement les communautés. Si les politiques publiques sont la réponse aux demandes des populations en vue du développement, il est important de préciser qu'il existe une priorité dans la demande car le seul État ne peut pas tout réaliser en même temps et efficacement. À ce sujet, la Figure 5 montre les domaines de politiques publiques qui préoccupent le plus les populations et qui sont mises dans la balance du chantage politique entre 2006 et 2016 par les acteurs politiques.

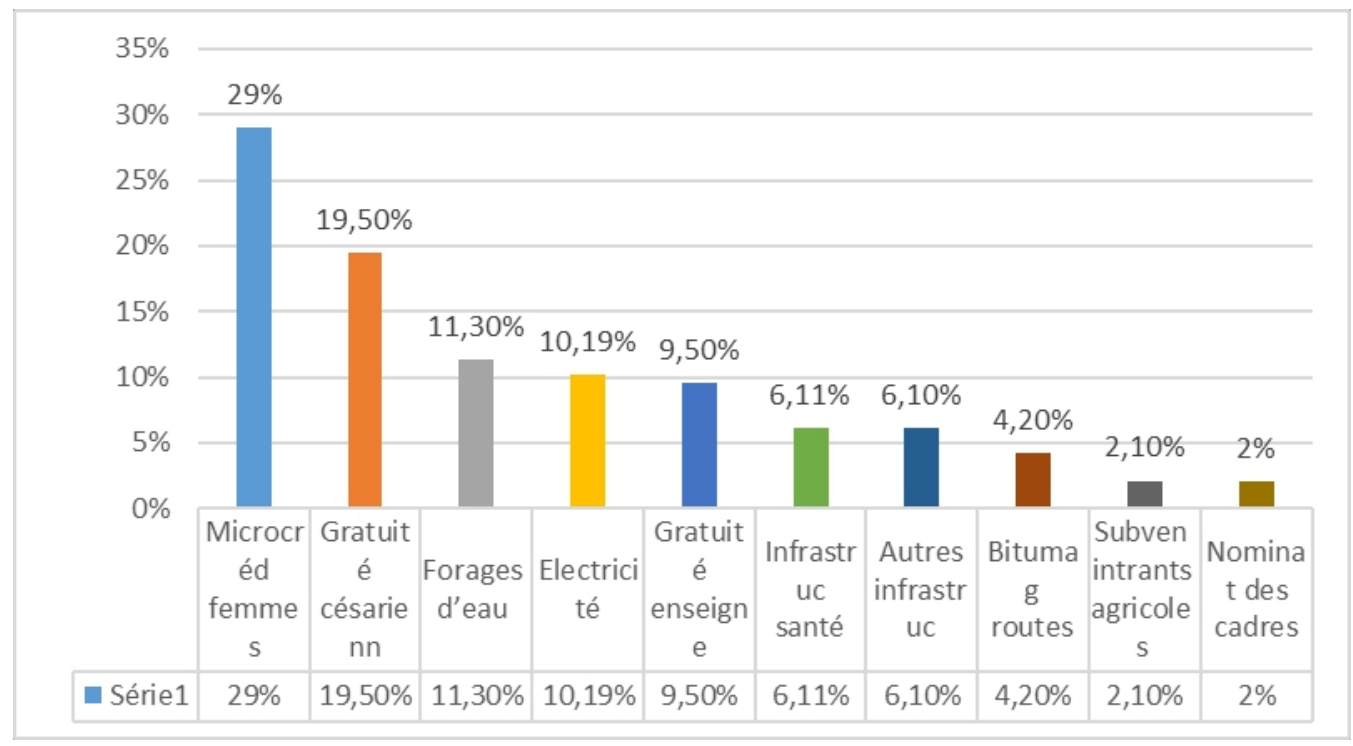

Figure 5. Catégories et tendances des politiques publiques de chantage politique Source : données d'enquête, janvier-juin 2018 
La Figure 5 montre clairement que les populations sont essentiellement préoccupées par 1) les microcrédits aux femmes $(29 \%)$, 2) la gratuité de la césarienne $(19,5 \%)$ et 3$)$ l'eau $(11,30 \%)$ qui sont des besoins vitaux pour le développement et des secteurs d'importance capitale. En revanche, le bitumage des routes $(4,20 \%)$, la subvention des intrants agricoles $(2,10 \%)$ et la nomination des cadres sont de moindre importance, pas en matière de développement, mais pour les populations lorsqu'il s'agit de formuler des demandes quant au développement. Tous les programmes de gratuité attirent particulièrement les électeurs parce qu'ils croient que par ce truchement ils bénéficient de la manne nationale et sont donc pris en compte dans le partage de la richesse nationale.

\subsubsection{Politisation de la mise à l'agenda politique}

Dans le processus de l'action publique, la mise à l'agenda politique est un acte capital de grande importance. Payre définit la «mise à l'agenda » en ces termes : «Elle [mise à l'agenda politique] est le processus démocratique par lequel les populations expriment leurs besoins tout en faisant le lobbying pour qu'ils soient pris en compte dans l'action publique » (Payre 2013 : 7). Pour qu'un problème social soit mis à l'agenda politique, il suit un processus bien précis :1) le problème se pose de façon récurrente, 2) le problème est très grave, 3) les acteurs concernés / victimes se mobilisent et donnent de la voix, 4) la médiatisation rend visible et perceptible le problème, 5) le lobbying et la pression sociale font prendre conscience aux autorités et décideurs en vue de l'élaboration des politiques publiques qui ne visent qu' à produire le développement depuis la base.

En effet, le problème qui se pose est que la mise à l'agenda politique entre 2006 et 2016 ne suit pas textuellement ce processus. Il a été observé des problèmes pertinents et préoccupants sur lesquels les populations ont fait un lobbying pressant mais cela n'a pas retenu l'attention des pouvoirs publics. Par contre, l'action publique s'est étendue sur des problèmes de moindre importance non demandés par personne mais pris en compte par l'action gouvernementale. C'est par exemple le cas de la gratuité de l'enseignement supérieur, la création du Fond National pour la Promotion de l'Emploi et de l'Entrepreneuriat des Jeunes (FNJEEP) ou le Service Militaire d'Intérêt National (SMIN) qui n'ont été nullement demandés. Les résultats obtenus dans chacune de ces politiques en sont très éloquents : 1) l'enseignement supérieur a baissé de qualité là où les acteurs bénéficiaires pouvaient légèrement contribuer pour être plus exigeants, 2) le Fond National pour la Promotion de l'Emploi et de l'Entrepreneuriat des Jeunes (FNJEEP) n'a pas pu atteindre les objectifs qu'il s'est fixé. Non seulement les emplois n'ont pas été créés mais les crédits cédés n'ont jamais été recouvrés à $25 \%$ et 3 ) le 
Service Militaire d'Intérêt National (SMIN) n'a pas duré suite aux problèmes liés à son financement et aux polémiques politiques qu'il a suscitées.

De 2006 à 2016, la mise à l'agenda politique était caractérisée par deux faits principaux. Premièrement, pour le moindre problème, les acteurs politiques suscitent la marche des populations qui scandent le nom du Chef de l'État. Ensuite, le Gouvernement même prend des initiatives très peu pertinentes en termes de demandes exprimées depuis la base. Il se dégage comme sentiment général que les autorités publiques agissent dans le seul but de plaire aux électeurs.

\section{Conclusion}

L'opérationnalisation des politiques publiques au Bénin de 2006 à 2016 n'a pas été un acte simple et direct. Le fait politique s'est emparé de tout et même de l'action publique du développement. C'est en cela que l'opérationnalisation des politiques publiques a été largement influencée par la négociation politique et la marchandisation politique, ce qui a engendré des conséquences.

En définitive, il est important de retenir que la négociation politique est un acte de portée appréciable en ce qui concerne la prise et la conservation du pouvoir politique. Pour ce faire, la négociation politique se met en œuvre à travers la recherche et l'affirmation des termes de négociation qui sont l'initiation des mesures de gratuités diverses, la facilitation de l'accès à l'eau potable, la construction d'infrastructures de santé, la construction d'infrastructures d'éducation, l'initiation des microcrédits aux femmes, la construction d'infrastructures de transport, le renforcement de la sécurité publique, la création d'emplois par le secteur agricole, la promotion de l'artisanat et de la transformation et l'ouverture des pistes rurales de desserte. Ces termes s'affirment par l'entremise des canaux de négociation politique tels que les lobbys religieux, les lobbys ethniques et régionaux, les lobbys des affaires, les lobbys des associations diverses, les lobbys familiaux et les lobbys corporatistes. Ces divers lobbys influencent négativement le processus de mise à l'agenda politique.

Parlant de la marchandisation politique, elle a lieu à travers le marché des politiques publiques et le marché des retombées politiques. En cela, les marchés publics et les affaires, les postes et placements politiques et les politiques publiques de développement sont les principaux offres et produits du marché politique. À ce niveau, il faut préciser que les politiques publiques n'ont pas la même importance pour chaque cible d'acteurs votant. Pour les populations à la base, les politiques publiques représentent tout. Elles représentent peu pour les élites politiques et ne représentent pas grand chose pour les hommes d'affaires qui ne sont préoccupés que par leurs affaires et intérêts personnels. Les politiques publiques sont les créateurs de 
développement. De même, le chantage politique fait partie des stratégies politiques auxquelles les acteurs politiques, à la recherche du pouvoir politique, font recours pour atteindre leurs objectifs.

Etant donné que la mise à l'agenda politique a été fortement politisée et que donc l'élaboration et la réalisation des politiques publiques tâche légitime et normale de tout pouvoir public, il est fondé de chercher à savoir si l'action publique pourrait être ainsi efficace: l'efficacité des politiques publiques dépend-t-elle de la seule objectivité politique?

\section{References :}

1. Bako, A. (1995). Démocratie et logique du terroir au Bénin. In : Politique africaine - Le Bénin, $n^{\circ} 59$. Paris : Karthala, pp $7-24$.

2. Banegas, R. (1998). Marchandisation du vote, citoyenneté consolidation démocratique au Bénin. In : Politique africaine - des élections comme les autres, $n^{\circ}$ 69. Paris, Karthala, 1998, pp $75-87$.

3. Banegas, R. (2000). La démocratie est-elle un produit d'exportation en Afrique ? L'exemple du Bénin. In: Christophe Jaffrelot (Dir.), Démocratie d'ailleurs. Paris : Karthala.

4. Bourdieu, P. (1981). La représentation politique : éléments pour une théorie du champ politique. In : Actes de la Recherche en Sciences Sociales, $n^{\circ} 37$. Paris : Savoirs scientifiques.

5. Braud, P. (1996).L'Emotion en politique. Paris : Presses de SciencesPo.

6. Braud, P. (2003). La démocratie politique. Paris : Seuil.

7. Champagne, P. (2001). Faire l'opinion : le nouveau jeu politique. Paris : Minuit.

8. Chevalier, J. (2005). Le service public. Que sais-je ? Paris : PUF.

9. Courty, G. (2006). Les Groupes d'intérêts. Paris : La Découverte.

10. Danioue, J. R. (1997). Afrique : l'unité de mesure démocratique. Paris, L'Harmattan.

11. Duverger, M. (2000). Sociologie de la politique. Gallimard, Université de Paris I.

12. Genieys, W. (2011). Sociologie politique des élites. Paris : A. Colin (Col., U).

13. Genieys, W. (2012). Gouvernement et action publique. Paris : Presses de Sciences Po (P.F.N.S.P.)

14. Institut National de la Statistique et de l'Analyse Economique (INSAE), République du Bénin (2014).4 ${ }^{e m e}$ Recensement Général de la Population et de l'Habitat (RGPH4). Cotonou: Editions Publication nationale.

15. Lapierre, J.-W. (2002). L'analyse des systèmes politiques. Collection le Politologue, Université de Nice, France. 
16. Muller, P. (2000). L'analyse cognitive des politiques : vers une sociologie politique de l'action publique. In: Revue française de science politique, 50 (2), p. 189-207.

17. Payre, R. (2013).Socio-histoire de l'action publique. Paris : La découverte.

18. Polin, R. (2007). L'obligation politique. Volume III, Paris : L'Harmattan.

\section{References Webographiques}

19. Centre d'Analyse Politique (CAP). Les acteurs programmatiques et le changement : plusieurs cas d'interactions en France et en Europe. Disponible sur www.dunod.com(page consultée le 28 mai 2017).

20. Dupy-Dery, M. L'analyse empirique des acteurs programmatiques : comment combiner méthodes de la sociologie des élites et de la sociologie de l'action publique? Disponible sur www.savoirspolitiques.info(page consultée le 13 avril 2018). 